\title{
Deep Observations with the Parkes 21-cm Multibeam System
}

\author{
M. J. Disney, P. J. Boyce, G. D. Banks, \\ R. F. Minchin and A. E. Wright \\ University of Wales, Cardiff, PO Box 913, Cardiff CF2 3YB, UK \\ mjd@astro.cf.ac.uk, pjb@astro.cf.ac.uk, gdb@astro.cf.ac.uk, awright@atnf.csiro.au \\ Received 1998 November 2, accepted 1999 February 8
}

\begin{abstract}
We report on a preliminary analysis of a $5600 \mathrm{sec}$ per point survey of 32 square degrees in Centaurus, carried out with the Parkes 13-beam system. The signal-to-noise ratio is found to improve as $\sqrt{t_{\mathrm{obs}}}$ for the whole integration. We have detected $102 \mathrm{Hi}$ sources between +250 and $+12,700 \mathrm{~km} \mathrm{~s}^{-1}$ either by eye or by using the new galaxy-finding algorithm PICASSO. Over half of these are new $\mathrm{H}_{\mathrm{I}}$ detections. Around a dozen of these are not associated with catalogued galaxies and, in two of these cases, we have not identified an optical counterpart on the Digitized Sky Survey. Arguments are put forward to explain why deep integrations are needed to find low surface brightness objects.
\end{abstract}

Keywords: galaxies: distances and redshifts — galaxies: luminosity functions galaxies: mass function — galaxies: statistics

\section{Why Go Deep?}

It is worth remembering that HIPASS is a shallow survey, with only $450 \mathrm{sec}$ integrations per point, and this may severely limit what we can find with the survey (Staveley-Smith et al. 1996). Therefore, it was always intended that much deeper observations would be made of limited areas of sky, though it was never certain how long such integrations could be profitably carried on, i.e. for how long the signal-to-noise ratio would continue to improve as $\sqrt{t}$ (Disney \& Banks 1997). We report here, on behalf of the DEEP team, some results from our early experiments.

Reasons for going deep are:

(a) To find intrinsically fainter dwarf galaxies. HIPASS (Banks et al. 1999) is capable of finding $\geq 10^{7} M_{\odot}$ of $\mathrm{HI}$ in a dwarf at a distance of 3.5 Mpc. In view of the present controversy over the faint end of the luminosity function (Phillipps et al. 1998; Trentham 1997) it is desirable to go deeper, particularly in nearby groups.

(b) We should expect that low surface brightness objects will generally have low Hi column densitiesand this is born out by observations (Bothun, Impey \& McGaugh 1997). However, system noise puts a limit to the lowest column density attainable by any radio telescope, irrespective of size, i.e.

$N_{\mathrm{HI}} \geq 10^{18} T_{\mathrm{sys}} \sqrt{\Delta V\left(\mathrm{~km} \mathrm{~s}^{-1}\right) / t_{\mathrm{obs}}(\mathrm{s})} \quad\left(\mathrm{cm}^{-2}\right)$ (e.g. Disney \& Banks 1997) and $N_{\mathrm{HI}}$ and surface brightness $\mu_{\mathrm{B}}$ (in blue mag per square arcsec) will be related through

$$
N_{\mathrm{HI}} \sim 10^{20}\left(M_{\mathrm{HI}} / L_{\mathrm{B}}\right) 10^{0 \cdot 4\left(27-\mu_{\mathrm{B}}\right)} .
$$

Thus the HIPASS survey would appear to have a column density limit $\left(T_{s} \simeq 23 \mathrm{~K}, \Delta V \simeq 150 \mathrm{~km}\right.$ $\mathrm{s}^{-1}$ ) of around $2 \times 10^{19} \mathrm{~cm}^{-2}$, which corresponds to a mean $\mathrm{SB}$ (if $M_{\mathrm{HI}} / L_{\mathrm{B}} \simeq 0.5$ ) of $28 \mathrm{~B} \mu$ over the whole hydrogen radius. If the $\mathrm{HI}_{\mathrm{I}}$ radius is twice the optical (van Zee, Haynes \& Giovanelli 1995) and if most of the light from the exponential disk comes from within four optical scalelengths then the central $\mathrm{SB}, \mu_{0}(\mathrm{~B})$, of a galaxy with $28 \mathrm{~B} \mu$ as defined above will be about $24 \mathrm{~B} \mu$. Thus we cannot expect to find many LSBGs with HIPASS. We need to go 1-2 magnitudes fainter, i.e to integrate for 6 to 36 times as long.

(c) A rather different way to approach this is to ask what we would have to do to find optically invisible objects in $\mathrm{HI}$ :

to detect in HI:

$$
\text { distance } d<d_{\max }\left(M_{\mathrm{HI}}\right) \sim\left(M_{\mathrm{HI}} / f_{\min }\right)^{\frac{1}{2}} \text {; }
$$

not to detect in optical:

$$
\text { distance } d>d_{\max }\left(L_{\mathrm{B}}\right) \sim\left(L_{\mathrm{B}} / l_{\min }\right)^{\frac{1}{2}},
$$

where $f_{\min }$ and $l_{\min }$ are the minimum detectable HI and optical fluxes respectively. Combining these: for an invisible $\mathrm{HI}$ detection 
$\frac{M_{\mathrm{HI}}}{L_{\mathrm{B}}}>\frac{f_{\min }(\mathrm{HI})}{l_{\min }(\mathrm{opt})} \quad$ (independent of distance) .

Now we can calibrate (3) for the Multibeam system, using our recent HIPASS survey of the Cen-A group $(d=3 \cdot 5 \mathrm{Mpc})$ (Banks et al. 1998) where we found $M_{\min }=10^{7} M_{\odot}, t_{\mathrm{obs}}=450 \mathrm{~s}$ and the faintest optical identification at $m_{\mathrm{B}}=19^{\mathrm{m}}$. We can thus construct Table 1 for the minimum $M_{\mathrm{HI}} / L_{\mathrm{B}}$ at which we could find invisible objects in different surveys.

Table 1. Minimum $M_{\mathrm{HI}} / L_{\mathrm{B}}$ ratios for optically invisible galaxies to be detected in different Parkes Multibeam surveys

\begin{tabular}{lccc}
\hline \multicolumn{1}{c}{ Survey } & \multicolumn{3}{c}{$\left(M_{\mathrm{HI}}\right) /\left(L_{\mathrm{B}}\right) \odot$} \\
& Normal galaxy & LSBG & VLSBG \\
\hline HIPASS $(450 \mathrm{~s})$ & 25 & $2 \cdot 5$ & $0 \cdot 6$ \\
$5 \times$ HIPASS & 11 & 1 & $0 \cdot 25$ \\
$12 \cdot 5 \times$ HIPASS & 7 & $0 \cdot 7$ & $0 \cdot 2$ \\
$25 \times$ HIPASS & 5 & $0 \cdot 5$ & $0 \cdot 1$ \\
\hline
\end{tabular}

The reason for the three different columns in Table 1 is that low SB galaxies show only part of their light above the sky. Thus a LSBG in the table is assumed to show only $10 \%$, the VLSBG only $2 \cdot 5 \%$, whereas the normal galaxy is assumed to show all (not of course strictly true).

Table 1 makes it clear that to find objects with the Multibeam which are not on the Digital Sky Survey and yet have $M_{\mathrm{HI}} / L_{\mathrm{B}}$ values that are not too extreme $(\leq 1)$, it is necessary to observe for at least five times longer than HIPASS.

\section{Noise in the Deep}

Given the novel nature of the multibeam system and the active scanning mode in which data are taken, there was no certainty that the noise would decrease with integration time as predicted. We have carried out an experimental survey to a depth of $12.5 \times$ HIPASS, i.e. $5600 \mathrm{sec}$ per beam, in a $4 \times 8$ degree region of Centaurus, taking all the data at night. The signal-to-noise in most parts of the frame (Figure 1) increases with $\sqrt{t_{\text {obs }}}$ throughout. The main sources of noise appear to be background continuum objects and characteristic long-wavelength ripples (caused by standing waves between the focus box and the

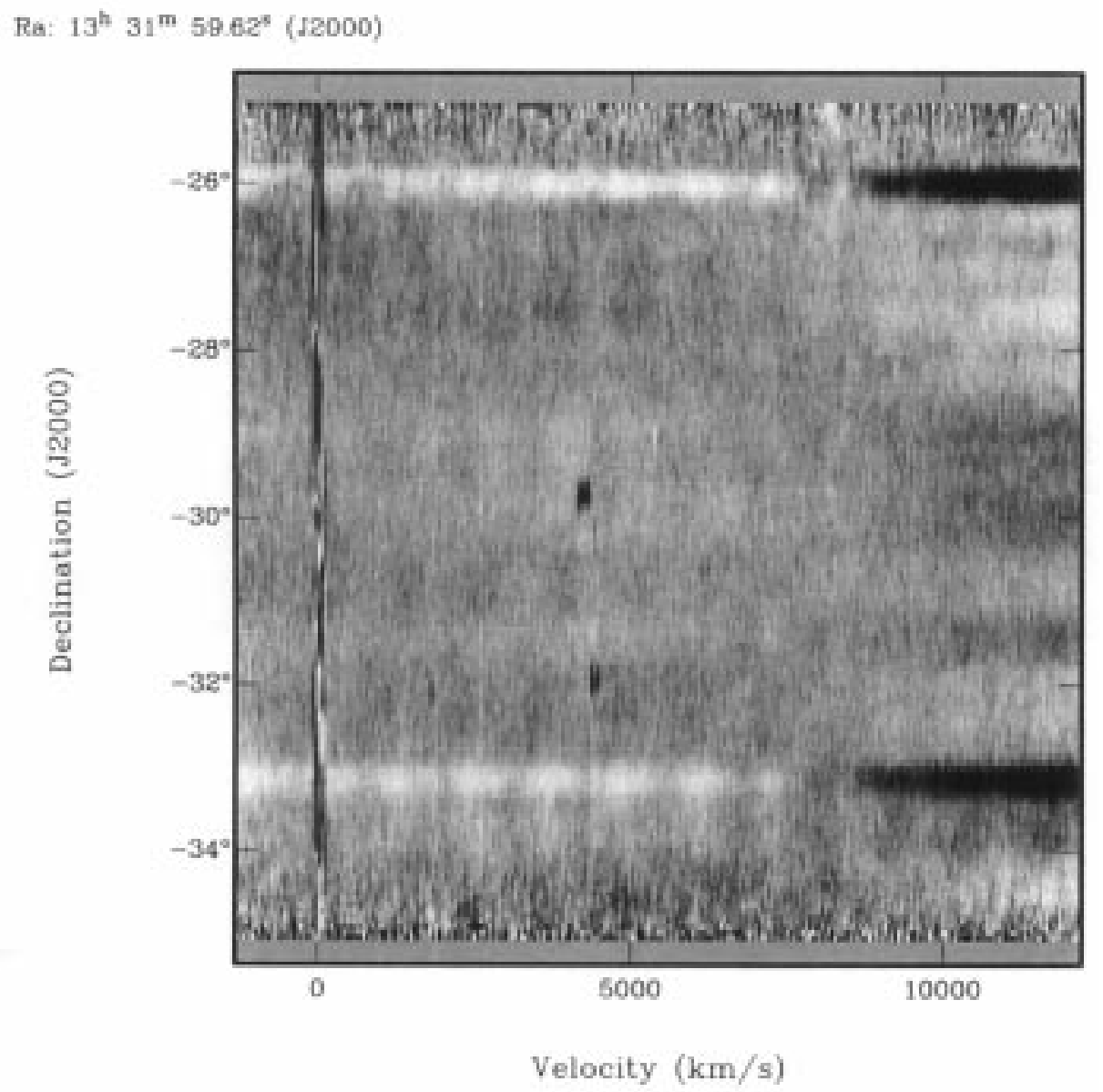

Figure 1-A portion of the Deep Cen survey cube with velocity in the horizontal direction, declination upwards. Note the Galactic Hi emission at zero velocity on the left, continuum sources at declinations of -26 and -33 , a real source at declination -30 and velocity around $4000 \mathrm{~km} \mathrm{~s}^{-1}$, edge noise at the top and bottom and the long baseline ripple with period $\sim 1000 \mathrm{~km} \mathrm{~s}^{-1}$. 

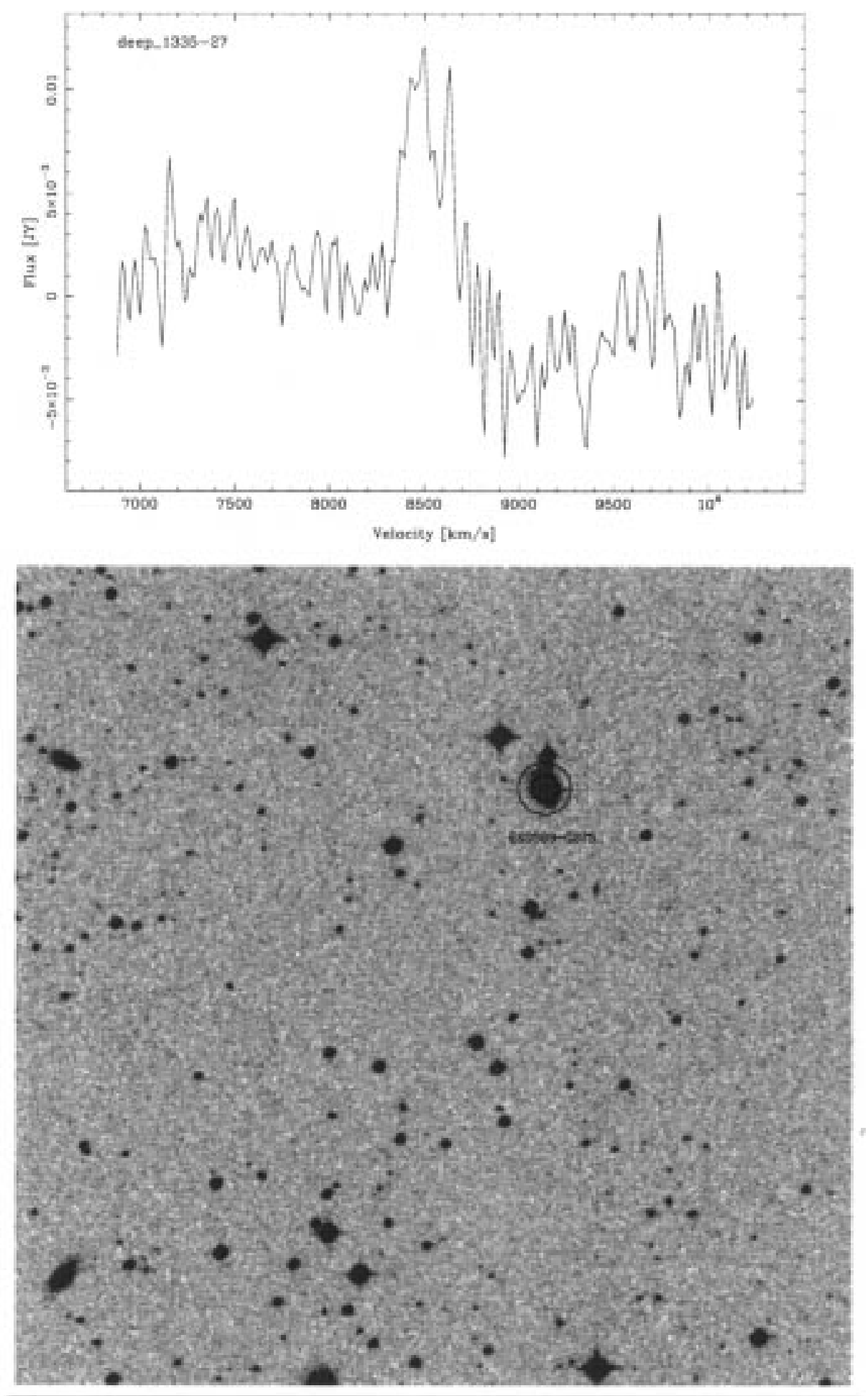

Figure 2-Hi spectrum and DSS image of one of the unassociated galaxies. The encircled object (marked on the DSS image) is the only galaxy within $5^{\prime}$ and has a velocity of $>12,000 \mathrm{~km} \mathrm{~s}^{-1}$.

surface of the dish) which look nothing like real $21-\mathrm{cm}$ sources. Thus there is hope of 'cleaning up' such cubes and going deeper still.

\section{Early Results}

We have searched the $8 \times 4$ degree cube by eye, and then again using PICASSO (father of cubism), an automatic galaxy-finding algorithm we have developed at Cardiff (Minchin 1999, present issue p. 12). By and large the two searches agree, though not perfectly. As a result we found a total of $10221-\mathrm{cm}$ sources in the cube (to a flux limit of $1 \mathrm{Jy} \mathrm{km} \mathrm{s}{ }^{-1}$ ). This is a conservative lower limit to the number we expect to find eventually and note 
that the edges of the cube will be noisier due to the survey strategy. Since the number of detected sources expected per unit area should go as $t_{\mathrm{obs}}^{3 / 4}$ this implies the number of detections for the whole sky in HIPASS to be $102 \times(40,000) / 32 \times(12 \cdot 5)^{-3 / 4}$ $\sim 20,000$. This, however, is a very rough estimate because of clustering statistics.

Of the 102 sources found, 41 were identified with catalogued galaxies with previously determined redshifts. For most of the remaining 61 sources, there is a catalogued galaxy without a measured redshift lying within $4^{\prime}$ of the fitted Hi position.

However, there are around a dozen sources for which no such association could be made. Studies of the Digitized Sky Survey fields around these sources revealed possible optical counterparts in most cases. However, there are still two for which no counterpart has been identified. Figure 2 shows the spectrum of one of these objects along with an image of the surrounding DSS field. We are undertaking CCD imaging of these candidate very low surface brightness galaxies.

\section{Future Work}

To summarise:

(1) We have been awarded time to extend our survey of this area to $23 \times$ HIPASS integration time.

(2) We have surveyed an area in Sculptor $(8 \times 8$ sq. deg.) to $5 \times$ HIPASS integration time. These data are currently being analysed.
(3) With the aid of Quentin Parker we are studying Tech-pan films of the Cen-A area to look for dimmer counterparts.

(4) We are presently carrying out follow-up CCD observations of the 'invisibles'.

(5) We are carrying out simulations to improve and validate the automatic galaxy-finding algorithm.

(6) We are experimenting with ways of cleaning cubes before sending them to the 'galaxy finder'.

\section{Acknowledgments}

Other members of the DEEP consortium include Ian Stewart, Mark Price, Ron Ekers, Ray Haynes, Erwin de Blok, Ken Freeman, Helmut Jerjen, Pat Knezek, David Malin, Mary Putman, Brad Gibson, Dan Zambonini and Quentin Parker. GB and RFM acknowledge the support of PPARC post-graduate research awards.

\section{References}

Banks, G. D., et al. 1998, 'New galaxies discovered in the first blind survey of the Centaurus A group', ApJ, submitted

Bothun, G. D., Impey, C., \& McGaugh, S. S. 1997, PASP, 109,745

Disney, M. J., \& Banks, G. D. 1997, PASA, 14, 69

Minchin, R. F. 1999, PASA, 16, 12

Phillipps, S., Parker, Q. A., Schwarzenberg, J. M., \& Jones, J. B. 1998, ApJ, 493, L59

Quintana, H., Ramirex, A., Melnick, J., Raychaudnury, S., \& Slezak, E. 1995, AJ, 110, 463

Staveley-Smith, L., et al. 1996, PASA, 13, 243

Trentham, N. 1997, MNRAS, 286, 133

van Zee, L., Haynes, M. P., \& Giovanelli, R. 1995, AJ, 109, 990 